\title{
Business Process Improvement Framework and Representational Support
}

\author{
Azeem Lodhi, Veit Köppen, and Gunter Saake \\ Department of Technical and Business Information Systems, \\ Faculty of Computer Science, \\ University of Magdeburg, Germany. \\ azeem, veit, saake@iti.cs.uni-magdeburg.de
}

\begin{abstract}
Business process management and improvement are vital for enterprises in competitive environments. Understanding of a process is a pre-requisite and important step for improvement. Interaction between humans, computers, and business objects provide excellent opportunities for knowledge extraction. However, the specification of a framework is required for business process improvement, which extends from data collection, analytical methods, storage, and representation of knowledge. The process models conceived for information system development are not sufficient for post execution analysis and improvement. In this paper, we specify such a framework briefly and focus on providing representational support for business process improvement. The main objective is to improve the overall improvement process by providing enriched graphical process models. Furthermore, we use a case study to explain the proposed usage and extensions of an existing modeling language for business process improvement.
\end{abstract}

\section{Introduction}

Business world is competitive where enterprises compete with one another to get the market share. Enterprises try to improve their existing processes in order to provide new or better services. Before making any improvements into existing operations, first, we need to understand how processes are executed in the enterprise. We should also find out the connections at various levels like inter and intra organizational level. For example, at a lower level between resources and activities whereas on higher levels between business units like sales, manufacturing, purchasing, and other supplies. Organizational decisions like maintaining stocks, maximum processing time of orders and others are important factors for an enterprise's success.

Computers are widely used in business as it ranges from handheld devices to main servers in order to run businesses. Human interacts with business objects (physical materials), organizational resources, and computer applications in order to run daily operations of enterprises. Information systems play an important role and record the interaction between humans, business objects, and applications during the execution of processes. These interaction records are useful to 
make improvement in overall businesses in case of either business process structure, organizational resource structure, or human-computer interaction (HCI). Analysis of a business process helps to identify deficiencies in existing processes.

Process mining techniques use those records to extract knowledge about business processes [1]. Similarly, application usage mining (AUM) [2] attempts to improve the design and structure of applications for improved interaction. However, a specification of a framework is required which will help to improve the existing processes. This framework should encompass data collection, analysis, and representation of results. Different questions regarding the framework are important like how the data is extracted from log files and converted for analysis. What are existing approaches in this context?

Business process modeling is important for overall understanding and improvement. Most of the research in business process modeling is focused on alignment of information technology with business processes [3]. Limited research is carried out to represent process knowledge through business process models for improvement [4]. Currently, information is represented with key performance indicators (KPIs), statistics, or visualizations (e.g., pie charts or histograms) which is too abstract and does not provide process details to business analysts. Similarly, current modeling approaches do not provide details where interactions can be further improved. Therefore, a graphical representation is very important factor for evaluation and knowledge representation for business process improvement [5].

In this paper, we address these issues and specify a framework for business process improvement. Business process context, stakeholders, and their participation is discussed in the next seciton. We briefly explain different components of framework and steps involved in business process improvement in Sect. 3. We also mention researchers' contributions in order to solve different issues related with components of the framework. After specification of an improvement framework, we shift our focus to the main contribution which is representational support for improvement in Sect. 4. From a human-computer interaction viewpoint, we also provide directions where business process modeling can be improved with intelligent human-computer interaction support for business process management. We also propose the usage and extension of an existing modeling language for business process improvement with the help of a case study. In the end, we summarize this paper and provide an outlook in Sect. 5.

\section{Business Process Context}

Customers' demands are fulfilled by enterprises which do business for several reasons, like satisfying stakeholders (executives, employees, and customers), monetary gain, or increase in reputation. In order to achieve these goals, business operations are carried out in a specific way. This specific way is called business process and governed by policies (rules and conditions). Different events occur (something "happens") in processes which usually have a cause (trigger like a request from the customer) or an impact (result of an activity) [6]. In response to 
these events, organizational resources perform operations on inputs and transform them into defined outputs. In this way, the final output is generated in an enterprise. The fulfilment of enterprise's goal is measured by metrics that evaluates the performance of a business process and its involved elements. This context of a business process is abstractly represented in the Fig. 1.

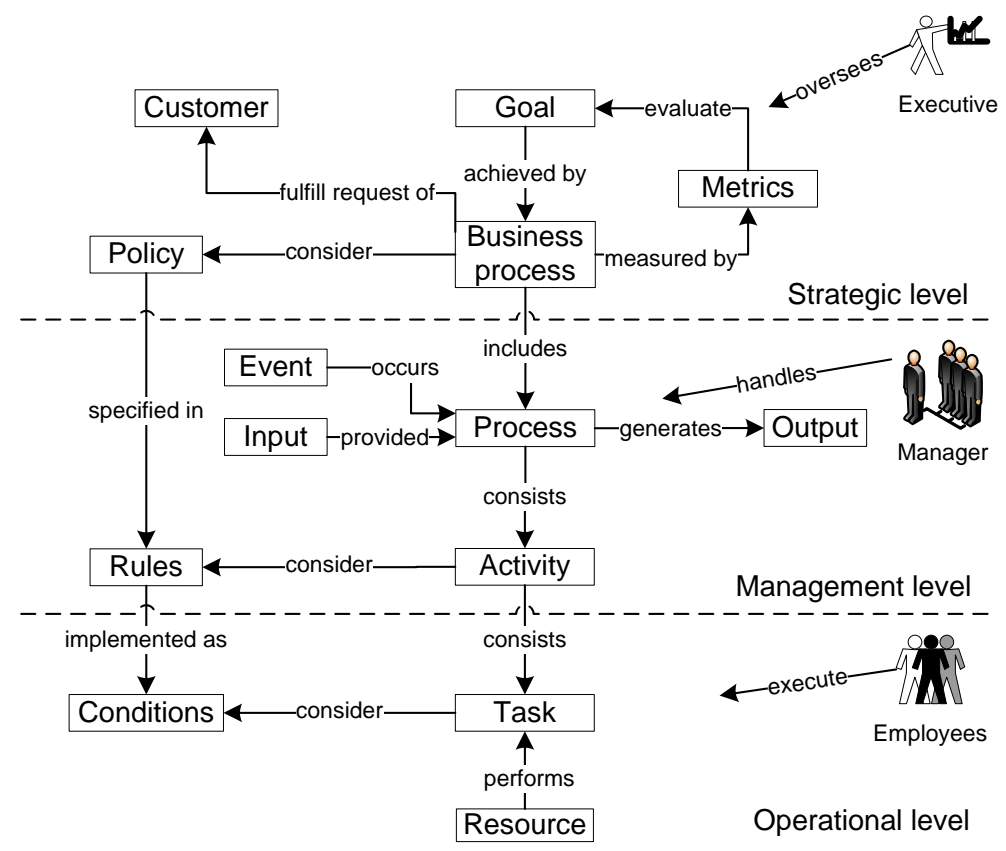

Figure 1: Business process meta model in different layers

Different stakeholders are involved in business processes at different levels. These stakeholders work on a business process at different levels. Therefore, they have different requirements and expectations from business process models. In the following, we list some of the stakeholders, levels, and their interaction with business process elements.

- Levels: Strategic level, management level, operational level

- Stakeholders: Executive, manager, employee

- Process: Business process, process, activity, task

At a strategic level, executives define goals and objectives of an enterprise. They view a business process as a black box and are concerned with overall output, profits, new acquisitions, and mergers. From a human-computer interaction (HCI) viewpoint, aggregated reports and statistics on handheld devices would be sufficient in case of remoteness. Managers, domain experts, and process managers are concerned with operations of enterprise and management of 
business processes. Tabletops, smart boards and other extended displays would be fruitful in analyses. Employees execute business process operations with a view of taking inputs, performing defined actions based on certain conditions using organizational resources, and then forwarding the result of those actions to other activities. We show some of the involve stakeholders at different levels in business process (BP) context meta model in Figure 1.

Elements define at one level are propagated to the other levels as well. For example, the goal of an enterprise is propagated as a process goal at the management level and continued as an activity and task goal to the lower levels. Similarly, metrics are computed on operational and management levels as well. Therefore, an analytical improvement framework and its representational support have to accommodate the requirements of different stakeholders.

\section{Framework for Improvement}

Business process improvement is important for enterprises due to competition and changes in markets like customer demands and technological changes. Business process improvement is a challenging task because of the complexity to carry out the changes in processes. Enterprises find it difficult to decide from where to start and what changes should be carried out for improvement. Complexity in business process is due to various factors like inter-dependencies between activities, stakeholders, involved elements, their attributes, and applications. Research in business process improvement needs to address these complexity issues and provide support to decide which action should be taken for business process improvement.

In order to provide the required support for business process improvement, a complete framework for analysis is required to be specified. This framework should address issues like data collection from information systems, its computation, representation in business process models, defining steps for improvement, and mechanism to carry out the changes. An abstract picture of the overall framework is shown in the Fig. 2.

Different techniques related with process mining [1] attempt to solve the issues related with data collection $[7,8]$ and its conversion $[9,10]$. Similarly in [11], author proposes a data format for recording business events. This BPAF (Business Process Analytics Format) helps in correlating and aggregating business events from different systems to one location. In [12], authors store data from logs into a data warehouse for different analysis. Therefore, data is collected from $\log$ files of information system and converted into a suitable format for analysis (this data is sometimes referred as process trace data).

Afterwards, several techniques (like process mining algorithms [13-15] and business process intelligence [12]) are applied on process trace data to extract knowledge. Application usage mining [2] techniques analyze user's behavior with applications and attempt to improve the interaction between applications, humans and their working. Similarly, other techniques can also be applied like balance scorecards and other process metrics. A knowledge base is also required 


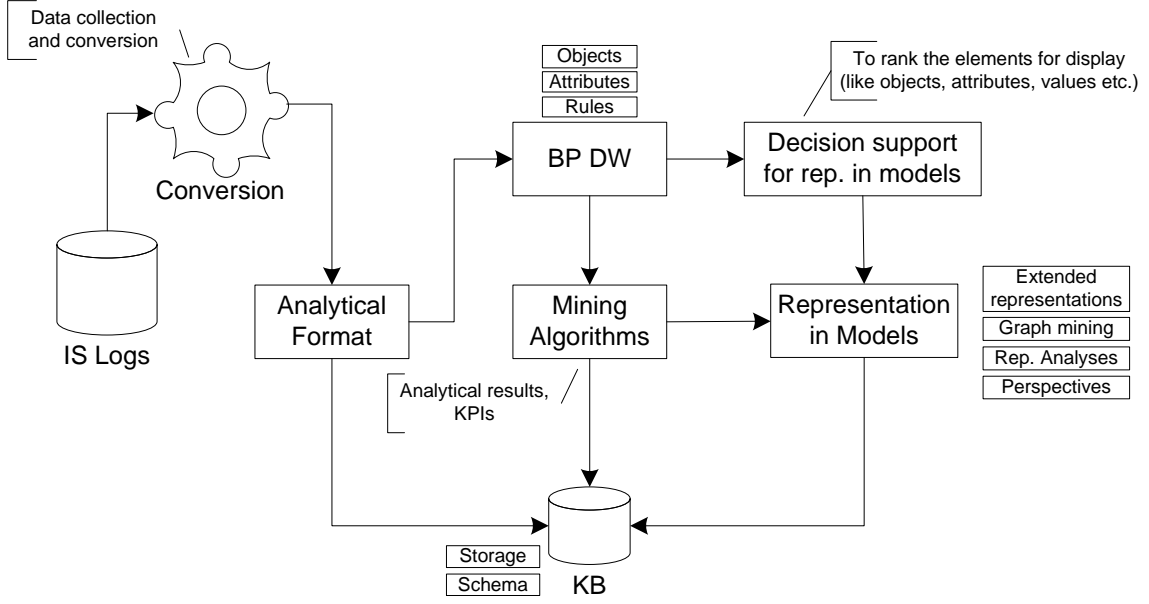

Figure 2: Framework for business process improvement and analysis

to store the gain information and knowledge from analysis techniques. Several issues are needed to be further investigated like what would be the schema design or format for storage? How the knowledge will be reflected as a company's best practices or used in enterprise operations?

The extracted knowledge and information are represented in a graphical form for intuitive understanding. For this purpose, depending on the stakeholders, certain information is filtered or provided with more details (context). Therefore, a decision support system is required which will rank the knowledge for representation with the context for stakeholders. This is also very important to reduce the cognitive load of users in graphical business process models while representing performance details and other knowledge.

Graphical representation of extracted knowledge and process trace data also provide an opportunity to apply different methods like graph mining [16]. Similarly, extended business process models help analysts to understand processes and identify deficiencies in business processes. We discuss the extended representation and benefits in the following section.

\section{Representational Support}

Graphical business process models are used to visualize the operations of a business process. Being graphical in nature, they are used for better understanding and analysis of business processes. They provide a medium of communication between stakeholders at different levels as described in Sect. 2. Business process modeling has increased the ability to understand business processes and to make rational decisions for organizing activities in a traceable and understandable way [17]. Business process modeling is used in different phases of a business 
process lifecycle, like, from identification of business processes to implementation in an information system [3].

Several gaps occur in business process lifecycle due to lack of communication, especially during the transition from one phase to another phase. Therefore, different models are proposed in literature to fulfill the requirements of stakeholders in business process lifecycle. For example, in planning phase Event-driven process chain (EPC) models [18], Flowcharts [19], and other models are used for communication. Similarly, for implementation into IT services different models are proposed, e.g., for software engineering different types of UML diagrams [20] exist. Therefore, different models and languages are devised to fulfill these gaps and improve the understanding of business processes.

Mostly, business process modeling is investigated for phases before its execution like analysis, design and implementation. Limited research is carried out to provide graphical modeling support for evaluation phase (including post execution analysis). In this section, we discuss how to provide the representational support for business process evaluation and improvement.

\subsection{Evaluation and Business Objects}

In evaluation or improvement of business processes, at a certain time, only specific elements (business objects) are considered in particular perspectives. For example, in organizational perspective resources of organizations are considered, like employees and machines. Similarly, in control flow perspective, sequence or collaboration of activities, and conditions are analyzed. These business objects have certain characteristics which define their state. Further characteristics of business objects become important when they get involved in business process (process executions) and interact with other objects. In different analyses, business objects are analyzed from various dimensions.

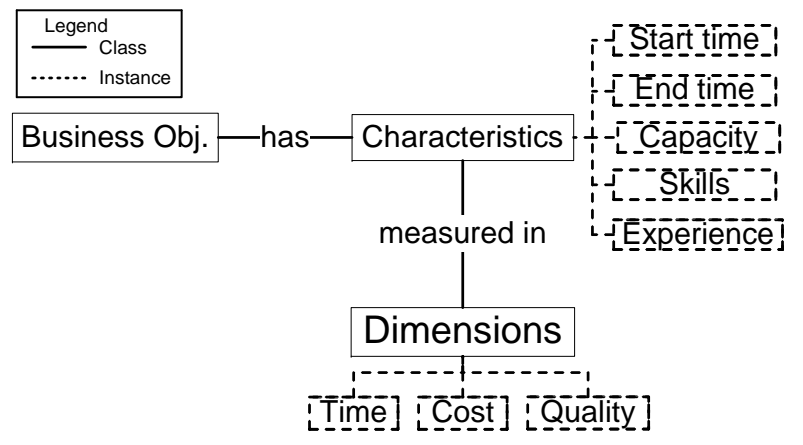

Figure 3: Business object characteristics and dimensions

Examples of these dimensions are time, cost, and quality. Different attributes of business objects and processes are analyzed under these dimensions. We show 
the relationship of business objects, characteristics, and dimension in the Fig. 3. We further discussed details of business objects and business processes in [5].

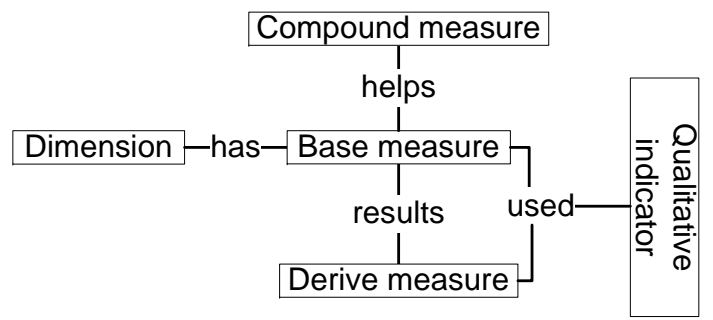

Figure 4: Evaluation meta model with base and derive measures

Evaluation of business process and business objects are carried out in different dimensions using base measures and derived measures. Base measures are main measurements from which other measurements are derived. For example, in time dimension the base measures are processing time and idle time. From base measures, different other measures like total time, minimum, maximum, and average time are derived. Compound measures are the measurements which are computed from different other measures like number of requests fulfilled in a particular time. Based on different measurements qualitative indicators are estimated like efficiency and quality as shown in the Fig. 4.

\subsection{Case Study}

Consider a small and simplified process given in Fig. 5 where five activities are executed to manufacture a product. In this process, first the parts are collected in activity A followed by activity B and activity $\mathrm{C}$ which are executed in parallel. These parallel activities assemble the specific parts of product independently from one another. After completion, the quality of assembled parts is checked in activity $\mathrm{D}$. If the assembled parts meet the quality requirements, then product is shipped in activity $\mathrm{E}$ and the process is completed. If the assembled parts do not fulfill a desired level of quality, then activity $\mathrm{F}$ is executed which disassembles the parts. Assuming that there is no loss during disassembly process, some part of the process has to be repeated again until desired level of quality is achieved. Therefore, activity $\mathrm{B}$ and activity $\mathrm{C}$ will be executed again. This process is modeled in the Fig. 5 using business process modeling notation (BPMN) [6].

Business process modeling notation (BPMN) is a standard defined by the Object Management Group (OMG) for modeling business processes. In BPMN specifications [6], BPMN graphical notations are divided into four basic categories. These categories are flow objects (for events, activities, and decision nodes), connecting objects (to connect flow objects), swimlanes (to represent process participants), and artifacts (for additional information). 


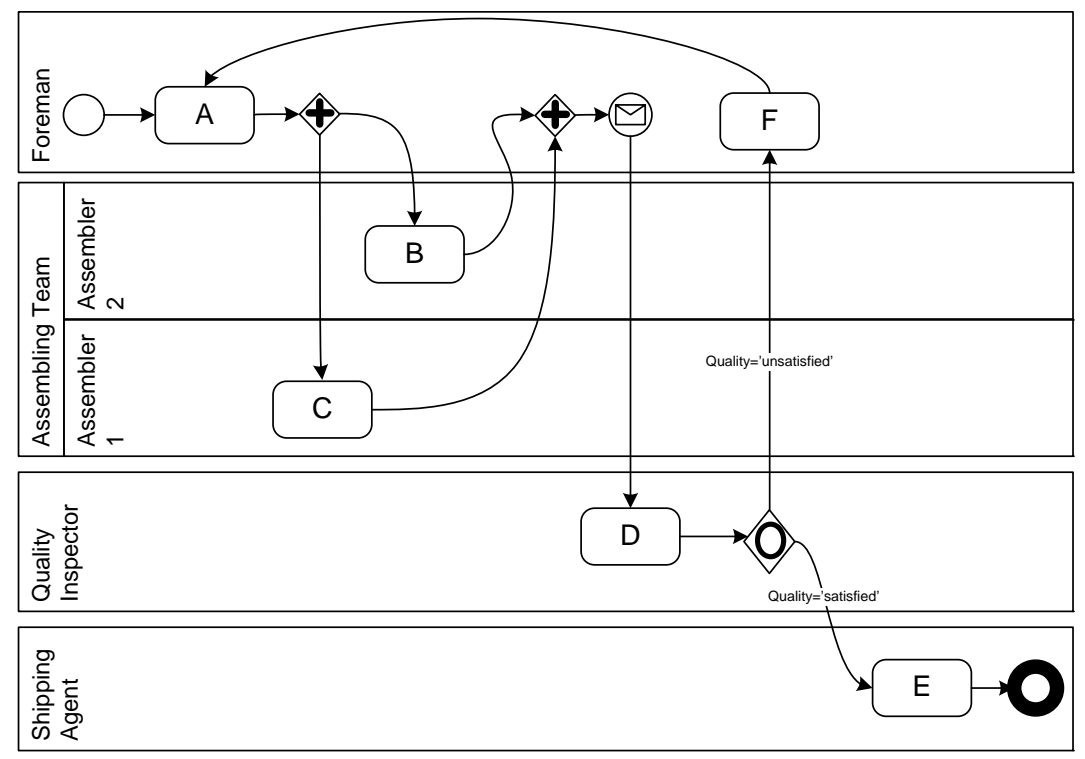

Figure 5: Product manufacturing example using BPMN

\subsection{Proposed Usage of BPMN Model for Evaluation}

Availability of analytical data and records of business objects in business process execution provide means to analyze processes more carefully. Here, we discuss two approaches for analysis using business process models and analytical data. In the first approach, we propose a method to use process models with analytical data for analyzing activities in different dimensions. In the second approach, we extend business process models with constructs to get a better understanding of process execution and their performance. These two approaches assist one to another for detailed business process analysis in order to find out the deficiencies and for process improvement.

Swimlanes as Dimension and Attributes: Mostly, in literature [6], Swimlanes (pool and lane) are used to represent organizational elements and their interaction during a business process. However, in this approach, first activities and involved elements are classified based on a particular dimension using analytical data. We recommend that only few classes should be defined for less cognitive loads of process models.

In our paper, we use Swimlanes (pools and lanes) to represent dimensions and different classes of these dimensions. Processes activities are aligned according to a selected dimension and its attributes (overall average values can be used for this classification) based on user defined threshold values.

We define three classes in cost dimension to arrange activities of processes and their involved elements in the Fig. 6. Similarly, different dimension attributes 


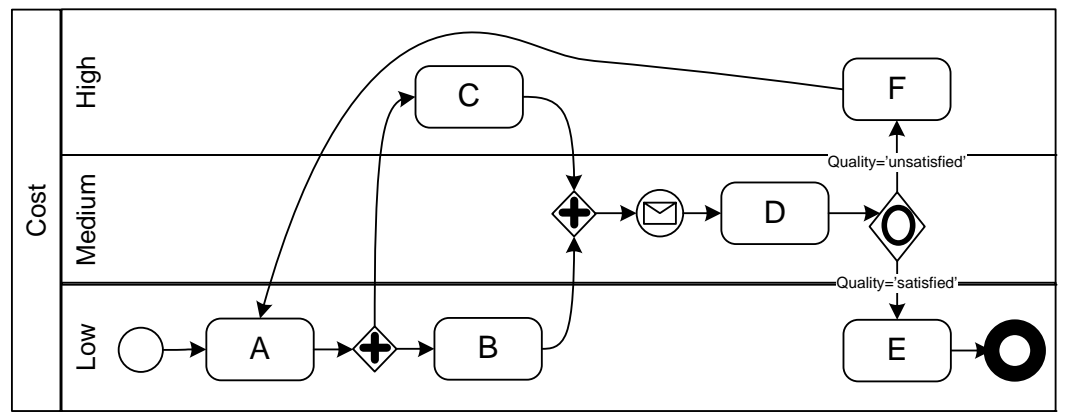

Figure 6: Process model representation based on costs

can also be defined as pools where lanes represent further classes of these attributes as shown in the Fig. 7. In Figure 7, the same activity is represented into two different attributes of time dimension. This Figure shows that the actual operating time would be very less but with high waiting time. For simplicity, we have not shown different other involved activities. Moreover, different dimensions and their attributes can be mixed with one to another for further business process analysis.

\subsection{Extending BPMN Models for Analysis}

Extended notations are also used to represent the participation of the different involved elements in process execution such as organizational resources. We propose the following extensions in BPMN for business process analysis and improvement.

Performance Based Classification and Colors in Representation: The executional data can be used to specify usage of which activities, organizational resources, and involved elements add more value to an enterprise. Different analyses provide this information in terms of cost and time. Based on this information, different colors can be assigned to activities, the process path of optimal execution, and involved elements. Similarly, the best practices process path of enterprise can also be colored.

Different colors can be used to indicate the effect of business objects like green for optimal cost, blue for optimal time, and red for non-optimal paths. Although, the relation of cost and time is not that simple as discussed in [21].

Colors in Process Paths: During execution, values of operational objects (inputs) changes by involved business objects. The change in values gives us details what operations are already performed and which path is taken. During analysis, different colors can also be used to represent the instance executional history, like which path instance has taken and at which particular stage a certain 


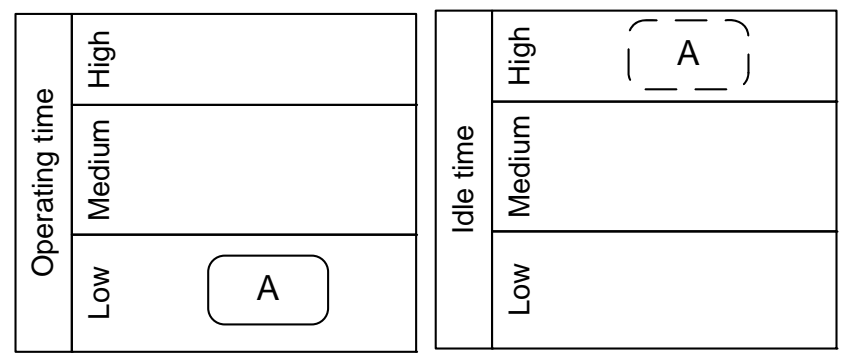

Figure 7: Process model in time dimension and its attributes

decision is taken. For this, such data can also be extracted from information systems logs or operational databases.

In Figure 8, we propose new symbols to represent different operational objects (like physical), organizational resources (if not represented in lanes/pool), and data objects with attributes. These symbols are necessary to provide more understanding of business scenarios and its corresponding process models. Similarly, the values of attributes are very important in case of business rules and conditions. Because, values of attributes play a major role in deciding the route of an instance. Based on these extended notations, we represent an extended business process model of our case study in the Fig. 8. The colors red, yellow, and green represents the elements with high, medium, and low cost respectively.

Depending on the granularity of detail, a business process may consist of many processes and activities which can range up to hundred and thousand. For process improvement, process managers are interested in details of these activities with extended representations which may increase the complexity of a business process model. Apart from the decision support system as discussed in Sect. 3, tabletops and smarts boards will be very useful for providing a full picture of a business process as compared with computer screens. Management of complex business process models and their representation in tabletops need further investigation. Similarly, tangible interfaces and tabletops can also be further investigated in order to provide help for training new employees with extended representation.

\section{$5 \quad$ Summary \& Outlook}

In this paper, we proposed a new business process improvement framework and its different components. We discussed researchers work related with different components and current challenges. We focused on representational component of improvement framework. We proposed a usage method and extension of BPMN for performance analysis which help to identify the deficiencies in a business process for improvement. In future, we plan to provide more extensions of BPMN and a complete case study involving different components of the im- 


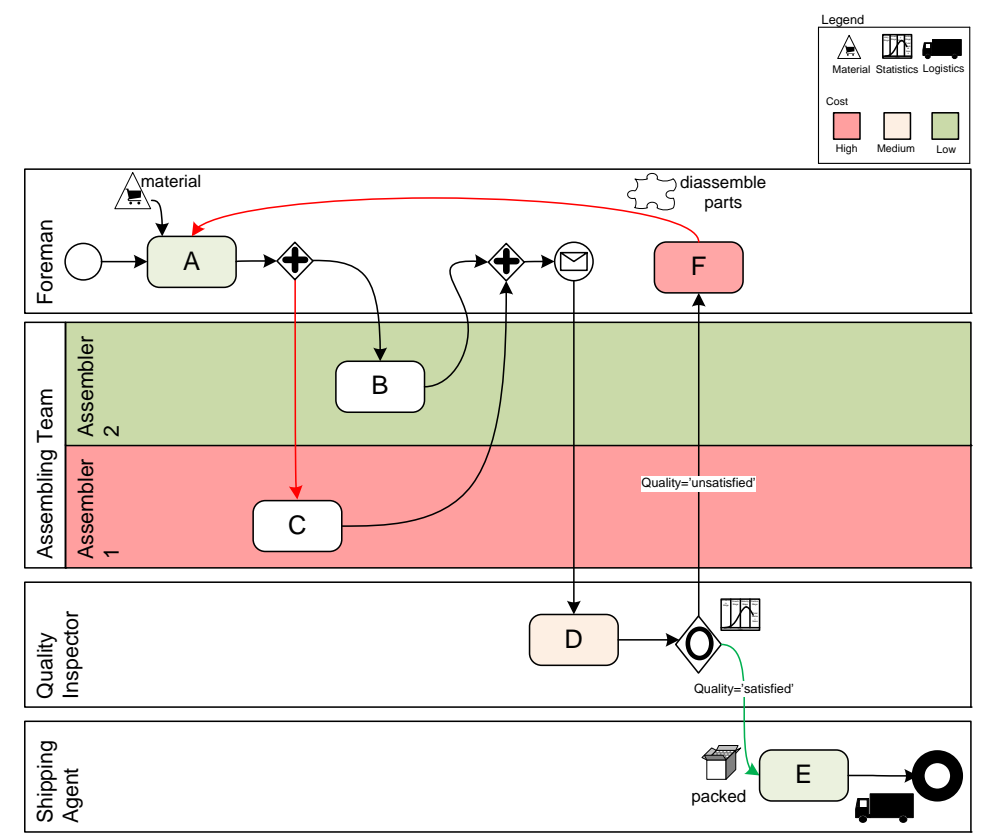

Figure 8: Performance based extended model with involved business objects

provement framework. We also plan to address open challenges introduced in this paper, like knowledge storage, better representation, and integration into business process models. Application of the proposed analytical framework in an industrial case studies is planned as well.

\section{Acknowledgment}

Azeem Lodhi is supported by a grant from the federal state of Saxony-Anhalt in Germany. This work is partially supported by the German Ministry of Education and Science (BMBF), within the ViERforES-II project No. 01IM10002B.

\section{References}

1. van der Aalst, W., Weijters, A.: Process mining: A research agenda. Computers in Industry 53 (2004) 231-244

2. Kassem, G., Rautenstrauch, C.: Application usage mining to improve enterprise workflows: ERP systems SAP R/3 as example. In Khosrow-Pour, M., ed.: Managing Modern Organizations Through Information Technology. Information Resources Management Association (2005) 358-362

3. Lodhi, A., Köppen, V., Saake, G.: Business process modeling: Active research areas and challenges. Technical Report 1, University of Magdeburg (2011) 
4. Vergidis, K., Tiwari, A., Majeed, B.: Business process analysis and optimization: Beyond reengineering. Systems, Man, and Cybernetics, Part C: Applications and Reviews, IEEE Transactions on 38(1) (2008) 69-82

5. Lodhi, A., Köppen, V., Saake, G.: Post execution analysis of business processes: Taxonomy and challenges. Technical Report 9, University of Magdeburg (2010)

6. BPMI.org, OMG: Business Process Modeling Notation Specification, Final Adopted Specification (Feburary 2006)

7. Khan, A., Lodhi, A., Köppen, V., Kassem, G., Saake, G.: Applying process mining in SOA environments. In Dan, A., Gittler, F., Toumani, F., eds.: Service-Oriented Computing ICSOC Service Wave 2009 Workshops. Volume 6275 of Lecture Notes in Computer Science. Springer (2010) 293-302

8. Ingvaldsen, J., Gulla, J.: Preprocessing support for large scale process mining of SAP transactions. Business Process Management Workshops 4928 (2008) 30-41

9. van Dongen, B., van der Aalst, W.: A meta model for process mining data. In Missikoff, M., Nicola, A.D., eds.: Proceedings of the Open Interop Workshop on Enterprise Modelling and Ontologies for Interoperability, Co-located with CAiSE. Volume 160 of CEUR Workshop Proceedings., Porto, Portugal, CEUR-WS.org (2005)

10. Günther, C., van der Aalst, W.: A generic import framework for process event logs. In Eder, J., Dustdar, S., eds.: Business Process Management Workshops, Workshop on Business Process Intelligence. Volume 4103., Springer (2006) 81-92

11. zur Muehlen, M.: Business process analytics format (BPAF) Document Number WFMC-TC-1015. Workflow Management Coalition USA (2008)

12. Grigori, D., Casati, F., Castellanos, M., Dayal, U., Sayal, M., Shan, M.C.: Business process intelligence. Computers in Industry 53(3) (April 2004) 321-343

13. Rozinat, A., van der Aalst, W.: Decision mining in ProM. In: International Conference on Business Process Management (BPM 2006). Volume 4102., Berlin, Germany, Springer-Verlag (2006) 420-425

14. van der Aalst, W.: Business alignment: Using process mining as a tool for delta analysis and conformance testing. Requirement Engineering 10(3) (2005) 198-211

15. van der Aalst, W., Reijers, H.A., Song, M.: Discovering social networks from event logs. Computer Supported Cooperative Work 14(6) (2005) 549-593

16. Lodhi, A., Kassem, G., Köppen, V., Saake, G.: Investigation of graph mining for business processes. In: Proceedings of The International Conference on Intelligence and Information Technology ICIIT. Volume 2., Lahore, Pakistan, IEEE Computer Society (Oct. 2010) 293-297

17. Cumberlidge, M.: Business Process Management with JBoss jBPM: A practical guide for business analysts. Packt Publishing (2007)

18. Scheer, A.W.: ARIS-Business Process Modeling. 2 edn. Springer-Verlag, New York, Inc. Secaucus, NJ, USA (1998)

19. IBM: Flowcharting techniques. Technical report, IBM Data Processing Techniques, Yorktown Heights, NY (1969)

20. Booch, G., Rumbaugh, J., Jacobson, I.: Unified Modeling Language User Guide. 2 edn. Addison-Wesley (May 2005)

21. Vullers, M.J., Kleingeld, P., Loosschilder, M., Reijers, H.A.: Performance measures to evaluate the impact of best practices. In: Proceedings of Workshops and Doctoral Consortium of the 19th International Conference on Advanced Information Systems Engineering (BPMDS), Tapir Academic Press Trondheim (2007) 359-368 